\title{
LA DESPERSONALIZACIÓN EN PSICOANÁLISIS
}

\author{
Rodrigo V. Abínzano ${ }^{1}$ \\ Universidad de Buenos Aires, Argentina. \\ abinzanopsi@gmail.com \\ ORCID: 0000-0002-7513-9707
}

DOI: 10.17533/udea.affs.v16n31a10

\section{Resumen}

El presente trabajo tiene como finalidad rastrear la genealogía y el arraigo que ha tenido la noción de despersonalización en psicoanálisis, específicamente en la obra de Jacques Lacan. Dicha noción, procedente de la tradición psiquiátrica, fue traída a colación en su enseñanza con fines variados, razón por la cual, en vías de poder cernirlo y darle un uso clínico, consideramos necesario repasar e interrogar lo que se ha elaborado en relación a ésta. En vías de ordenar nuestro proceder, establecemos la siguiente hoja de ruta: a) interrogar las menciones de despersonalización realizadas por Lacan en su Tesis de 1932;.) ubicar la noción en relación al final de análisis;) ubicar la noción en relación al concepto de fantasma y su vacilación.;) interrogar las referencias vinculadas a las perturbaciones del yo y lo especular.) establecer conclusiones y líneas de investigación futuras.

Palabras claves: despersonalización, Lacan, fantasma, final de análisis.

Recibido:20/10/2018 • Aprobado:22/04/2019

1 Psicoanalista. Lic. en Psicología (UBA). Miembro del Foro Analítico del Río de la Plata (FARP) y de la Internacional de los Foros del Campo Lacaniano (IFEPFCL). Becario de investigación UBACyT. Psicólogo clínico en C.S.M. n³ Arturo Ameghino (CABA). Docente de las cátedras "Psicología clínica de adultos y psicoterapia” (Cat. I) y "Clínica de las anorexias, bulimias y obesidad" (UBA). Enseñante colaborador del Colegio Clínico del Foro Analítico del Río de la Plata. Autor de Lecturas freudianas de la anorexia mental (Escabel, 2018). 


\section{Introducción}

Mediando el seminario de la angustia, Lacan se interroga por los bordes yoicos con la realidad, intentando ubicar cuál es la fenomenología que mejor da cuenta de dicho litoral. Luego de tomar a lo siniestro como el paradigma de la angustia -en tanto este concepto da cuenta, como ningún otro, de la perturbación a nivel de lo especular-, Lacan trae a colación una categoría proveniente del campo de la psiquiatría: la despersonalización (2009/1962-1963, p. 133). Establece allí tres puntos: el primero es que los fenómenos de despersonalización acompañan a la angustia; el segundo, que son los fenómenos más contrarios a la estructura del yo como tal; y el tercero, que el psicoanálisis no ha agrupado de modo sistemático dichos fenómenos.

Uno de los posibles interrogantes que se desprende de esta cita -especialmente en relación al último punto- es si otra disciplina -la psiquiatría, por ejemplo- sí lo ha hecho o lo ha realizado de modo menos ambiguo. De esto ya se han ocupado algunos autores (Napolitano et al., 2008), pensando las articulaciones posibles de la despersonalización con los tipos clínicos, tanto de neurosis como de psicosis e inclusive con la literatura, acentuando en su producción los aportes de la psiquiatría clásica como del psicoanálisis posterior a Freud.

La vía que no toman dichos autores -y que vertebra nuestra investigación- es la de interrogar las referencias hechas por Lacan en el correr de su obra, las cuales han sido variadas y permiten delimitar los usos posibles de esta noción, que podríamos inscribir en lo que Lacan llamó la "psiquiatrería” (Lacan, 2012/1971-1972, p. 18), ese cruce entre la psiquiatría y el psicoanálisis, donde se produce un saber que no es de uno ni del otro pero que nos permite avanzar en la labor clínica.

\section{La tesis de 1932: La despersonalización en la obra psiquiátrica de Lacan}

La obra psiquiátrica de Lacan contiene muchas menciones y elaboraciones en relación a la despersonalización. La finalidad de este 
apartado, dividido, a su vez, en tres subapartados, es circunscribir la pluralidad de coordenadas que hacen de antesala a la lectura psicoanalítica que se llevará a cabo a partir de la enseñanza formal, fechada por el propio Lacan en 1953. El punto de arribo principal será el movimiento dialéctico y relectura que hará de estos fenómenos con la categoría freudiana de lo Unheimlich, en el ya mencionado seminario dedicado a la angustia.

Retomando, en la tesis doctoral de Lacan encontramos referencias varias a la noción de despersonalización. Partiendo del concepto de personalidad, presenta la siguiente definición:

Un desarrollo biográfico, que definimos objetivamente por una evolución típica y por las relaciones de comprensión que en él se leen (...) una concepción de sí mismo con actitudes vitales y progreso dialéctico (...) y por una cierta tensión en las relaciones sociales, que definimos objetivamente por la autonomía pragmática de la conductas y los lazos de participación ética que en ella se reconocen. (Lacan, 1979/1932, p. 39).

Lacan toma dicha definición y la pone en diálogo con las posiciones de otras escuelas, en vías de no confundirlas. Comenzando el apartado dice: "la nuestra [la definición] no se funda en efecto: ni sobre el sentimiento de síntesis personal, tal como se le ve perturbado en los trastornos subjetivos de despersonalización, sentimiento que depende de mecanismos psico-orgánicos más estrechos" (Lacan, 1979/1932, p. 40). Si bien Lacan diferencia su definición de otras dos posturas, nos interesa particularmente el énfasis que hace en distinguirlo de la noción de despersonalización. Inclusive, en una nota a pie refiere que sería preferible sustituir por el término "trastornos de la personalización" el de "trastornos de la personalidad" y remite al lector a los trabajos de Hesnard (p. 40, n. 27).

\section{Los aportes de las escuelas alemana y francesa}

En el avance del estado de la cuestión de la tesis lacaniana, luego del relevamiento de los aportes de la escuela alemana a la psicogenia de las psicosis paranoicas, diferencia tres factores -carácter, vivencia y 
evolución- que deben vincularse con los términos en relación a los fenómenos de la personalidad que vimos antes:

En la determinación de la enfermedad encontramos una experiencia vivida ("vivencia") constituida por actitudes vitales asténicas y por la proyección sobre el plano de los valores éticos (progresos dialécticos) del sentimiento de insuficiencia concomitante. Este proceso ideo-afectivo se manifiesta en los fenómenos de represión y de inversión que constituyen el cuerpo de los síntomas; estos fenómenos son, esencialmente, una hipertrofia y una atipia de las imágenes ideales del yo en la consciencia; la evolución típica no muestra fenómenos de despersonalización. (Lacan, 1979/1932, p. 89, énfasis añadido).

Una vez más, Lacan se encarga de señalar que los fenómenos de despersonalización serían irrupciones atípicas en lo que hace al proceso normal de constitución de la personalidad. En este sentido, veremos que la crítica hecha por Lacan a la hora de considerar estos fenómenos dentro de la escuela alemana -recordemos la influencia que tenía en él la obra de Jaspers en este momento- también coincide con la crítica que les hará a algunos representantes de la escuela francesa.

El apartado dedicado al análisis de los autores franceses está vertebrado por la discusión en torno de las nociones de cenestesia, automatismo mental y sentimientos intelectuales, con un epílogo dedicado a la introducción de la noción de estructura en psicopatología a cargo de E. Minkowski. Como dijimos, la cenestesia -definida como "el conjunto de sensaciones propioceptivas e interoceptivas" (Lacan, 1979/1932, p. 115)- permanecen en un estado de sensaciones puras que no tienen percepción consciente en el estado de salud. T. Ribot, el padre del método psicopatológico, es quien ubica estas sensaciones difusas sobre la base del sentimiento psicológico del yo individual. Lacan expone su posición frente a dicha teorización:

Era tentador, en consecuencia, buscar en una alteración más o menos controlada de esa cenestesia el origen de los sentimientos mórbidos llamados de despersonalización, y a extender en seguida sus efectos a los sentimientos de inhibición y de depresión, a los sentimientos de influencia, así como a los sentimientos de extrañeza y de transformación del mundo exterior. O sea que en la base de 
todos estos fenómenos lo que había eran determinados trastornos de la cenestesia, cuya diversidad, por cierto, queda sin explicar. (p. 116, énfasis en el original).

La posición de Lacan es clara: no solo critica la lectura ribotiana que se ha llevado a cabo para explicar la fenomenología seriada, sino que también es crítico frente al hecho de que dicha concepción "seguía muy de moda" hacia 1900, gozando de todo su prestigio. A pesar de la posición de Lacan, hay esbozos de explicación en relación al porqué del sostén de dichas teorizaciones, como por ejemplo lo acorde que es pensar algunos delirios paranoicos y la ideación hipocondríaca desde la teoría cenestopática (p. 116).

Siguiendo con las concepciones de la escuela francesa, la teorización de los "sentimientos intelectuales" de P. Janet también contempla la problemática de la despersonalización. En lo que este autor denomina "sentimientos de incompletud" se encuentra una incompletud en la percepción de la propia persona, "o sea extrañeza del yo, desdoblamiento, despersonalización” (p. 119). Lacan se muestra ambiguo en el texto: en un primer momento refiere que el catálogo realizado por Janet tiene un "alto valor sugestivo"; luego valora la labor investigativa de su colega, especialmente en lo referente a su teoría de la psicastenia -recordemos las múltiples discusiones de éste con Freud-, y finalmente concluye que "esta colaboración es primitiva en relación con la aparición de los fenómenos mentales complejos" (p. 120).

\section{La noción de proceso y la despersonalización}

Arribamos al punto más importante en la tesis en relación a nuestra investigación: el apartado dedicado a la noción de proceso psíquico de Jaspers, lugar desde donde Lacan se situará para argumentar. Nos encontramos aquí con una de las distinciones más importantes en relación a estos fenómenos, porque desde este momento ya se sostiene que la despersonalización podrá ser pensada tanto en el plano de las neurosis como en el de las psicosis. Lacan toma el ejemplo del psi- 
quiatra Van Valkenburg, quien separa la etiología de la esfera afectiva y ubica, en el comienzo de las psicosis, la despersonalización:

Van Valkenburg aprecia al comienzo de las psicosis un sentimiento de despersonalización y toda una serie de pequeñas señales somáticas en las cuales se basa para admitir un proceso cerebral, no accesible todavía, por cierto, a la observación directa. Con todo, los casos que él aduce no parece que se puedan considerar como psicosis paranoicas verdaderas. (Lacan, 1979/1932, p. 128).

Párrafo seguido, Lacan introduce el método jasperiano como un "método sano que puede servir para aclarar los hechos" (p. 128) y cuyo concepto central es el de proceso psíquico, el cual se opone al de "desarrollo" de la personalidad. En relación a la referencia de Van Valkenburg, Lacan no critica tanto el hecho de que la despersonalización tenga lugar o no al comienzo de una psicosis, sino que no cree que pueda pensarse dentro del tipo paranoico.

Recordemos que Jaspers define la personalidad en su Psicopatología general como "el conjunto individualmente distinto y característico de las relaciones comprensibles de la vida psíquica" (Jaspers, 2014/1913, p. 478) y que la personalización, entendida como el proceso previo a que pueda aparecer un fenómeno de despersonalización, se sostiene en cuatro puntos: a) el hecho de que la conciencia de sí se opone a la de exterioridad y de los otros; b) el sentimiento de actividad; c) la conciencia de identidad (yo soy el mismo que antes y que siempre); d) la conciencia de unidad (yo no soy más que uno al mismo instante). Dice Jaspers: “Todos los procesos psíquicos y manifestaciones, en tanto que señalan por encima de sí una relación individual y totalmente comprensible, experimentada por el individuo con la conciencia de su particular yo íntimo, constituyen su personalidad" (p. 479).

Si bien la principal referencia de Lacan en la tesis es la teoría de K. Jaspers sobre el proceso psíquico, se pueden ver también influjos de conceptos psicoanalíticos como "superyó" o "libido", así como encontrar que en la bibliografía de la tesis hay incluidos diez textos de Freud (Lacan, 1979/1932, p. 332). Dicha aclaración nos parece pertinente, ya que cuando Lacan va a volver a hacer referencia 
al fenómeno de despersonalización -teniendo en cuenta que puede tener lugar tanto en las neurosis como en las psicosis- no es sin haber hecho un despliegue de la teoría libidinal y de traer a colación la nosografía freudiana (p. 234). En el desarrollo del apartado que denomina "Frutos del estudio del caso", la argumentación lacaniana refiere ciertos "esbozos de trastornos psíquicos" que son detectables como antecedentes. Dentro de estos habla de trastornos de la función sexual -frigidez, hiperexcitación-, de perversiones, episodios neuróticos obsesionales - obsesiones o fobias- y de sentimientos neuróticos de despersonalización (que llegan a veces al "sentimiento" o hasta "alucinaciones de desdoblamiento") (p. 245).

Unos párrafos más abajo, Lacan introduce una serie de elementos que, si bien tendrán algunas reformulaciones, mantendrá en su teoría de las psicosis: el mecanismo interpretativo diferenciado de la disociación esquizofrénica, la noción de "fenómeno elemental" y el valor "razonante" de la paranoia.

En relación al mecanismo interpretativo -quienes trabajamos analíticamente con sujetos psicóticos sabemos cuán importante es distinguir el mecanismo que opera en un fenómeno elemental-, Lacan dice que éste puede mostrar un abanico de trastornos perceptivos pero que tienen un carácter "harto razonante". Los clasifica: ilusiones de percepción, ilusiones de memoria, sentimientos de transformación del mundo exterior, fenómenos borrosos de despersonalización, pseudo-alucinaciones, e incluso alucinaciones episódicas (p. 246). Basándose en Kretschmer dice que los fenómenos alucinatorios sutiles no tienen valor diagnóstico ni pronóstico especial.

Hay un aspecto epistemológico que nos gustaría destacar en relación a la posición lacaniana dentro de la producción de la tesis. Allí afirma: "Hay que decir, por otra parte, que nuestra investigación de las psicosis toma el problema en el punto al que el psicoanálisis ha llegado en nuestros días" (p. 292); y a partir de allí Lacan delimita una "insuficiencia" dentro del psicoanálisis debido a la confusión que hay en relación a las fijaciones en el narcisismo o el autoerotismo. No obstante, es para remarcar el hecho de que es una coordenada delimitada por el psicoanálisis -especialmente por el concepto de narcisismo- desde donde hay que continuar la investigación en relación a las psicosis. 
En el marco de dicha crítica, Lacan hace una afirmación que podríamos pensar en consonancia con la realizada treinta años después, en el seminario sobre la angustia. Luego de interrogarse en relación a las fuentes de la libido, la naturaleza del yo como instancia psíquica y las nociones de preconsciente e inconsciente, refiere:

Sobre el valor económico mismo de los síntomas en que de manera más sólida se funda la teoría del narcicismo (síntomas de despersonalización, ideas hipocondríacas: ¿se trata aquí de hechos de sobrefijación o de desfijación libidinal? Es ésta cuestión sobre la cual las opiniones difieren de todo a todo). (1979/1932,p. 293).

El reclamo de Lacan a los analistas, de que no se habían ocupado de los fenómenos de despersonalización de manera sistemática, tiene su génesis en esta crítica que se sostiene durante treinta años y vuelve en el seminario dictado entre 1962 y 1963 . No es un dato menor que sea la libido la que esté en juego, ya que sabemos que Lacan hará una relectura del concepto energético freudiano para proponer la libido como órgano, un año después (Lacan, 2009/1964, pp. 194-208).

Resumiendo lo abordado en este apartado, vimos que las elaboraciones heterogéneas de las que parte Lacan sobre la despersonalización, no solo lo orientan por la vía transestructural, sino que también le permiten cernir una coordenada puntual: los momentos de aparición de angustia o pérdida de referencias por parte del sujeto.

\section{Acerca del ocaso imaginario: despersonalización y fin de análisis}

Hacia el final del seminario Los escritos técnicos de Freud, Lacan hace una referencia al fin del análisis. Recordemos que desde lo planteado por Freud en su escrito Die endliche und die unendliche Analyse, traducido como "Análisis terminable o interminable" el fin del análisis ha sido un obstáculo a ser interrogado por las distintas escuelas de psicoanálisis. El título freudiano original -en un sentido literal- habla de un análisis terminable y de uno interminable, o también de un análisis finito y uno infinito. El punto a destacar es que, para Lacan, 
ya desde esta época el análisis tiene un fin, un punto de culminación, un momento de concluir.

Mostrando una de las que sería su fuente de interrogación e influencia constante, la literatura de los místicos, Lacan evoca la obra de Angelus Silesius, el Peregrino querubínico y cita:

Zufall und Wesen

Mensch werde wesentlich; denn wann die Welt vergeht

So fält der Zufall weg, dasswesen dass besteht.

Este dístico se traduce así:

Contingencia y esencia

Hombre, deviene esencial: pues cuando el mundo pasa,

la contingencia se pierde y lo esencial subiste. (Lacan, 2009/19531954, p. 339).

Previo a esta cita, Lacan argumenta fuertemente contra la idea de que el análisis progrese hacia una ampliación del campo del ego, y a reconquistar fronteras desconocidas; nos dice que es un vuelco, un desplazamiento, un paso de minué ejecutado entre el ego y el id. Lo que constituye el análisis -refiere- es el acto de la palabra (p. 338). Luego de citar al místico comenta:

De esto se trata al fin un análisis; de un crepúsculo, de un ocaso imaginario del mundo, incluso de una experiencia que limita con la despersonalización. Es entonces cuando la contingencia cae -el accidente, el traumatismo, las dificultades de la historia-. Y entonces es ser el que llega a constituirse. (p. 339).

En primer lugar, es imposible no dar cuenta de la influencia de Heidegger en esta afirmación de Lacan. Como algunos autores ya han destacado en relación al fin de análisis como realización del ser (Balmes, 1999, p. 30) o lo que hace al fin de análisis y al tiempo de ser (López, 2011, p. 161), la despersonalización aquí no solo puede ser pensada en el campo de las psicosis y las neurosis; además es constitucional del final del proceso analítico, por lo cual, el fin de análisis 
para el analizante implica una experiencia que limita con la despersonalización.

En ese sentido, articulando con la afirmación que vimos más arriba, siendo la despersonalización el fenómeno de mayor extrañeza yoica (Lacan, 2009/1962-1963, p. 133), esta idea del fin de análisis no dista mucho de un fenómeno de extrañeza yoico. A su vez, tampoco dista en gran medida de la afirmación realizada a finales de 1964, donde el fin del análisis es llevar a mayor distancia el objeto a del I (acan, 2009/1964, p. 281).

\section{Vacilación fantasmática y despersonalización}

En el análisis que Lacan realiza de Hamlet en El deseo y su interpretación, la despersonalización es traída a colación en vías de ubicar ciertos fenómenos en los límites del fantasma y sus vacilaciones. Es así que Lacan hace referencia a la siguiente escena de la tragedia shakesperiana: Hamlet había finalizado el diálogo en el cual el espíritu de su padre le revela la verdad acerca de su asesinato, pidiéndole venganza. Ofelia está en su cuarto cosiendo cuando Hamlet aparece "con tal doliente expresión en el semblante como si hubiera escapado del infierno para contar los horrores" (Shakespeare, 2003/1609, p. 120). Polonio exclama que eso se debía al amor que el príncipe sentía por su hija, lo que Lacan ubica como una vacilación de Hamlet con su objeto. Sin necesidad de forzarlo, Lacan dice que lo que le sucede "podemos considerarlo emparentado con esos períodos en que irrumpe una desorganización subjetiva, cualquiera sea" (Lacan, 2012/19581959, p. 354). Continúa:

Un fenómeno semejante tiene lugar en la medida en que algo vacila en el fantasma y hace aparecer sus componentes, reunidos en lo que se denomina una experiencia de despersonalización. He aquí lo que se manifiesta en esos síntomas. Los límites imaginarios entre el sujeto y el objeto llegan a transformarse y pasan al orden de lo llamado fantástico, en el sentido estricto del término. (p. 354).

Si teníamos en la cita del Seminario 1 una referencia a la despersonalización en relación al final del análisis, aquí encontramos una 
vinculada a la vacilación fantasmática, donde Lacan les adjudica el estatuto de "síntomas", ubicando lo "fantástico" en los límites imaginarios entre el sujeto y el objeto. Esta referencia se complementa cuando, unos párrafos luego, Lacan trae a colación lo Unheimlich, no enlazado a fenómenos inconscientes -los cuales advendrán una vez que el síntoma sea puesto en forma-, sino que remiten a un momento previo de desequilibrio del fantasma.

Lacan continúa:

Eso es justo lo que ocurre cuando algo de la estructura imaginaria del fantasma, $(\$<>a)$, logra comunicar con lo que llega mucho más fácilmente al nivel del mensaje, $\mathrm{s}(\mathrm{A})$, a saber, la imagen del otro, i(a), dado que es mi propio yo que se sitúa por debajo del mensaje. Autores como Federn señalan con mucha sutileza las correlaciones necesarias entre el sentimiento del cuerpo propio y la extrañeza de lo que le ocurre al sujeto en cierta crisis, cierta ruptura, cierto detrimento del objeto en el nivel especificado como (\$<>a). (p. 354).

La referencia lacaniana apunta al esquema del grafo, donde el mensaje no pasa por el circuito que le implica cierto rodeo, sino que se presenta de modo directo; es mi propio yo -dice Lacan- que se sitúa por debajo del mensaje. Un punto también a destacar es el comentario positivo de los trabajos de P. Federn; sabemos que Lacan se valió de los desarrollos de los postfreudianos, en su mayoría, para criticarlos; esto es notorio en lo que hace a la clínica de las psicosis, de donde Lacan no toma aparentemente nada. ¿Podría ser lo dicho por Federn una excepción?

\section{Federn con Lacan: despersonalización y fronteras del yo}

Si recordamos, en su libro La psicología del yo y las psicosis, Federn dedica un capítulo entero al problema de la despersonalización ${ }^{2}(\mathrm{Fe}-$

2 Sobre este punto véase especialmente el artículo de E. Soengas y J. Martín “Los fenómenos de despersonalización: los antecedentes", incluido en el ya citado Napolitano et al., 2008, pp.17-38. Un punto a destacar, por lo llamativo, es que Lacan no menciona en el correr de su enseñanza los trabajos de P. Schilder, quien trabajó en profundidad los fenómenos de despersonalización. 
dern, 1984/1952, pp. 266-286.). Su exposición comienza destacando la necesidad de ubicar la noción de "enfermedades del yo", diferenciándola de otras como "individuo", "persona" o "personalidad integrada" (p. 266). Para delimitar síntomas yoicos específicos hay que ir al campo de las perturbaciones psíquicas del yo, donde el autor introduce dos perturbaciones yoicas monosintomáticas: la despersonalización y el extrañamiento; ambas pueden ser parciales o totales, por lo que refiere a un carácter gradual para hablar de la variedad y dice que el término "extrañamiento" es más preciso que el de "despersonalización", para el que prefiere "atonía del yo", evidenciando una pérdida de la firmeza de la instancia yoica. Dice: "puede definirse la despersonalización como la vivencia subjetiva del desquicio del propio yo" (p. 268). No obstante, no habría una distinción clara entre extrañamiento y despersonalización, ya que ambas se entremezclan al presentarse en el campo perceptivo. Para este autor, el extrañamiento y la despersonalización no son ni neurosis ni psicosis, sino que son una perturbación cualitativa, al modo de las neurosis actuales (p. 272); pueden ser la antesala de cualquiera de las antes nombradas, dependiendo el nivel de perturbación de la investidura.

Un punto muy importante para nuestro desarrollo es la paradoja que destaca Federn con estos fenómenos:

Todos los fenómenos de extrañamiento y de despersonalización muestran una y la misma paradoja. Aunque están alteradas las percepciones, apercepciones y propiocepciones, la inteligencia y los sentidos, así como las habilidades y conocimientos y la capacidad de adaptación del individuo permanecen incólumes. (1984/1952, p. 269).

El fenómeno de despersonalización no podría ser considerado un síndrome o síntoma confusional, ya que las habilidades generales no están afectadas: quien lo padece no pierde ninguna actividad vinculada al campo de la voluntad, la memoria o la atención; y unas líneas más abajo, Federn también descarta una etiopatogenia orgánica ( $\mathrm{p}$. 270).

Una apreciación que nos parece acertada tiene que ver con cuál sería la explicación de lo que sucede, ya que todos los sentidos fun- 
cionan con exactitud, “ya sea que la perturbación esté en la visión, la audición o el pensar vinculado con los objetos, el resultado es siempre la falta de familiaridad" (p. 270). Es allí que este autor ubica la perturbación en las fronteras yoicas, definidas como "el órgano sensorial para toda apercepción de la realidad". Toma el ejemplo freudiano clásico del déjà $v^{3}$ como el paradigma del extrañamiento. Federn enfatiza en que "ningún objeto es privado de su realidad" sino que lo que se pierde -como vimos- es la "familiaridad" (p. 279), otro punto que lo acerca a la conceptualización freudiana de lo siniestro (Freud, 2007/1919, pp. 215-252).

En lo que hace a la clínica de las psicosis, la despersonalización queda como un fenómeno a ser tenido en cuenta como posible indicador de una esquizofrenia y la desrealización del lado de las psicosis depresivas. Un aporte importante es que Federn concluye que la despersonalización puede tener estatuto tanto de síntoma como de trastorno, donde en el primer caso probablemente acompañe una neurosis y en el segundo se juegue en el terreno de las psicosis. Hacia el final enumera una serie de problemas nosológicos, posición argumentativa esclarecedora, ya que da cuenta de Federn como investigador, no dando por cerrada su teorización. Si bien excedería a nuestro propósito en este trabajo, los interrogantes planteados por el autor giran en torno de las investiduras yoicas y los grados de perturbación del aparato ante la aparición del fenómeno.

\section{La irrupción en lo especular: despersonalización, estructura del yo y desconocimiento}

La referencia más citada en relación al tema de la despersonalización dentro del psicoanálisis lacaniano es la que se encuentran en el seminario de la angustia, mencionada al comenzar este trabajo. Luego de seguir el hilo argumentativo de Lacan en relación al tema, se esclarece

3 La mayoría de los autores que han leído los fenómenos de despersonalización y desrealización en la obra freudiana siempre hacen mención tanto a los déjà $v u$ como a la "perturbación" de la memoria sufrida por Freud en el texto del recuerdo de la Acrópolis (2007/1901; 2007/1936, pp. 209-222). 
un poco lo dicho allí y se nos permite abordar luego lo que dice en seminarios posteriores.

En la clase del 23 de enero de 1963, hablando sobre los fenómenos de borde en el yo, dice:

Lo encontramos de nuevo muy claramente en fenómenos que se hallan entre los más conocidos que acompañan la angustia, los designados como fenómenos de despersonalización. Son precisamente los fenómenos más contrarios a la estructura del yo como tal. Esto suscita para nosotros una cuestión que no podremos evitar, la de situar auténticamente la despersonalización, en la medida en que bajo esta rúbrica, si se ven las divergencias de los autores, se agrupan los fenómenos de una forma ciertamente ambigua desde el punto de vista analítico. (Lacan, 2009/1962-1963, p. 133).

Retomando, esta cita tiene la particularidad de puntualizar al menos tres cuestiones: la primera, el hecho de que los fenómenos de despersonalización acompañan la angustia; la segunda, que son el fenómeno más contrario a la estructura del yo como tal; y la tercera, el psicoanálisis no ha hecho un agrupamiento sistemático o no ambiguo sobre el tema.

El hecho de que los fenómenos acompañen la angustia resuena con lo trabajado en relación al fin de análisis y la vacilación fantasmática: habría algo en ambos momentos que podría pensarse de manera homóloga, ya que hay un movimiento de los cimientos de la realidad, planteando inclusive dos momentos de pasajes. El momento de la vacilación, ligado a la irrupción de la angustia, posible antesala de la formulación de un síntoma analítico, y en el caso del fin de análisis, como un acto performativo, donde hay un pasaje de analizante a analista.

No termina de quedar claro a qué se refiere Lacan allí con "estructura del yo", ya que podría pensarse como una extrañeza que el fenómeno genera al yo como instancia unificada e ilusoria, o que implique una extrañeza a la estructura del yo en cuanto escindido, afectado por la Spaltung de manera estructural.

En el apartado anterior repasamos los desarrollos de un representante de la Ego Psychology, quien destaca para el yo "su fascinan- 
te rasgo de unidad" (Federn, 1984/1952, p. 281); esto, cotejado con el breve texto freudiano Die Ichspaltung im Abwehrvorgang, muestra un distanciamiento, ya que allí no solo el yo "esta desgarrado" por el proceso defensivo, sino que además dicho desagarro "nunca se reparará, sino que se hará más grande con el tiempo" (Freud, 2007/1940, pp. 275-276). Lacan claramente en esto es literalmente freudiano.

En lo que hace al último punto, el de la necesidad de ordenar y sistematizar el fenómeno, algunos autores se ocuparon de delimitarlo con los tipos clínicos y las estructuras (Napolitano et al., 2008) pero quedaba por hacer un rastreo de lo que el propio Lacan había dicho sobre el tema, desarrollo muy variado y con distintos puntos de encuentro a nivel conceptual.

Lacan continúa:

Fenomenológicamente, parece obvio que la despersonalización empieza con el no reconocimiento de la imagen especular. Todos saben hasta qué punto este hecho es palpable en la clínica, y con qué frecuencia es al no reconocerse en el espejo, o cualquier cosa análoga, cuando el sujeto empieza a ser presa de la vacilación despersonalizante. Pero esta fórmula que da cuenta del hecho no deja de ser insuficiente. Si lo que ve en el espejo es angustiante, es por no ser algo que pueda proponerse al reconocimiento del Otro. (Lacan, 2009/1962-1963, p. 134).

Volviendo a la constitución del estadio del espejo mediante el reconocimiento -imposible no escuchar ecos hegelianos- hay algo del no reconocimiento que genera una vacilación despersonalizante mismo término que usó para hablar de lo que pasa con el fantasma en el Seminario 6-. Advertidos, dicha fórmula -es como si Lacan hiciera un resumen de lo que vino articulando en relación a la despersonalización- es insuficiente, por lo cual introduce al reconocimiento del gran Otro; punto crucial y hasta ahora inédito en sus menciones de la despersonalización, porque queda a la luz que quien despersonaliza es el Otro, o más bien su ausencia de reconocimiento.

Lacan retomará su modelo paradigmático para pensar estos fenómenos, lo siniestro. Si Lacan toma una serie de categorías de la psiquiatría, como por ejemplo la de despersonalización, es para apropiarse de ellas mediante el psicoanálisis. El concepto de lo Unhei- 
mlich le permite hacer eso con el de despersonalización. Allí vemos que cuando el a no está velado por la imagen, se produce una irrupción de lo ominoso. Es también por el objeto, su inscripción o no como pérdida, que Lacan podrá establecer diferencias de estructuras que afectan el modo en que leamos el fenómeno de despersonalización.

\section{La clínica y los efectos objeto a: La despersonalización como crisis y pequeño mal}

Vimos en el apartado anterior los efectos a nivel de la imagen que tiene la introducción del objeto a. En Problemas cruciales para el psicoanálisis, Lacan avanza en esa empresa acentuando las características propias del objeto a, como por ejemplo el hecho de ser acósmico, de estar por fuera de la escena del mundo, y de producir efectos, tanto por su extracción como por su no extracción.

En relación a dicha diferenciación vinculada a las estructuras, Lacan se vale de un caso de Pearl King, del cual dice que: "es exigible que en lo que va a seguir, les diga lo que es un objeto a en la psicosis, la neurosis, perversión. Eso no es parecido" (Lacan, 1964-1965, clase del 3/2/1965). Se plantea, de este modo, una diferenciación a nivel estructural:

Pero hoy, quiero decirles cómo, en un analista seguramente sensible a su experiencia, el objeto a se les aparece; poco importa aquí que el caso con el cual ella promueve sus reflexiones sea un caso borderline, con cosas que se han hasta etiquetado: pequeño mal, a menos que eso no sea crisis de despersonalización. (Lacan, 19641965, clase del 3/2/1965).

Lacan ubica la despersonalización como un "pequeño mal" y como una crisis, en relación al caso de esta analista. Habla allí de "bordes psicóticos" que lindan con lo esquizoide, pero el punto importante es la diferenciación mediante el lugar que tiene el objeto a. Algunos autores, como Alomo, han hecho una lectura de este caso con esa orientación -diferenciar mediante el lugar del objeto el diagnóstico- proponiendo el caso de esta autora como “Un caso de esquizofre- 
nia tratado como si no lo fuera", a falta de "el buen corte" que debe hacer el analista (Alomo, 2012, pp. 90-92); hace eco con la afirmación realizada por Lacan en su seminario sobre la identificación de que "el corte hace a la estructura" (Lacan, 1961-1962, clase del 30/5/1962).

En lo que hace a nuestro tema, vemos que el fenómeno -aquí denominado como "crisis" - tiene carácter de "pequeño mal" en relación a otras presentaciones. Si bien Lacan no lo dice explícitamente, aquí, al parecer, sigue la afirmación hecha por Federn de que la despersonalización puede presentarse como una "leve crisis nerviosa" (Federn, 1984/1952, p. 279).

\section{Algunas conclusiones y posibles líneas de trabajo futuras}

En el presente trabajo hicimos un recorrido por la noción de despersonalización en la obra de J. Lacan. Para ello, en un primer momento, interrogamos las elaboraciones plasmadas en la tesis de 1932. Pudimos delimitar los aportes que hicieron tanto la escuela de psiquiatría francesa como la alemana para sus elaboraciones y la articulación hecha por Lacan mediante la noción de proceso psíquico de K. Jaspers.

En un segundo momento, abordamos la referencia hecha por Lacan en su seminario Los escritos técnicos de Freud, donde la despersonalización es traída a colación para pensar el fin de análisis. Posteriormente abordamos lo dicho en su seminario sobre El deseo y su interpretación, donde la despersonalización es abordada desde la vacilación del fantasma. En ese punto hicimos una lectura de los aportes de P. Federn, autor mencionado por Lacan y del cual pudimos extraer una serie de elementos para pensar dicha noción.

En un tercer momento fueron las referencias hechas por Lacan en el seminario sobre La angustia, donde el fenómeno acompaña dicho afecto y donde la despersonalización representa el grado de mayor diferencia con la estructura del yo. Vimos también que Lacan aborda, desde la categoría freudiana de lo siniestro, dicho fenómeno y nos permite -ya en el Seminario 12- pensar la despersonalización en una clínica diferencial del objeto, donde abordando un caso clínico trae 
a colación la categoría, pensándola como un "pequeño mal" o una "crisis".

A partir de ello podemos exponer las siguientes conclusiones:

Lacan se ocupó del fenómeno de despersonalización desde el comienzo de su práctica como psiquiatra. Ya en 1932 recriminó al corpus teórico del psicoanálisis no hacer una delimitación rigurosa de dicha categoría. Treinta años después volvería a afirmar lo mismo en el seminario de la angustia.

La despersonalización no puede ser pensada solamente como una categoría nosográfica y psicopatológica. Pueden ubicarse fenómenos de despersonalización tanto en las vacilaciones fantasmáticas como en el fin de análisis. También se podría agregar: y con muchas de las experiencias de los místicos, siguiendo los ejemplos que Lacan da en el Seminario 1.

La despersonalización es transestructural: esto no solo es una afirmación de Lacan, también lo han hecho muchos autores dentro del campo de la psiquiatría como del psicoanálisis.

La despersonalización no tiene un carácter uniforme y acompaña la angustia.

La despersonalización es el fenómeno más disímil de la estructura del yo. En ese sentido, la estructura del yo debe ser entendida como "unidad engañosa", a diferencia de lo que afirma de dicha instancia Federn.

Si bien Lacan toma el término de la psiquiatría, lo hace para apropiárselo mediante el psicoanálisis. El concepto de Unheimlich es el más pertinente para pensar lo que hace a la despersonalización. Lacan ya lo había anunciado en el Seminario 6 cuando dice que allí se desdibujan los límites entre el sujeto y el objeto y aparece lo "fantástico".

La despersonalización puede manifestarse en un déjà vu, en una perturbación a nivel de la memoria, en una perturbación al nivel de la imagen, así como en fenómenos que desdibujan los límites de la realidad y la confunden con el mundo onírico. 


\section{Referencias}

Alomo, M. (2012). La elección irónica. Estudios clínicos sobre la esquizofrenia. Buenos Aires, Argentina: Letra Viva.

Balmes, F. (2002/1999). Lo que Lacan dice del ser. Buenos Aires, Argentina: Amorrortu.

Federn, P. (1984/1952). La psicología del yo y las psicosis. Buenos Aires, Argentina: Amorrortu.

Freud, S. (2007/1901). Psicopatología de la vida cotidiana. En J. Strachey (Ed.), Obras Completas (J. L. Etcheverry, trad., Vol. VI, pp. 1-270). Buenos Aires, Argentina: Amorrortu.

Freud, S. (2007/1919). Lo ominoso. En J. Strachey (Ed.), Obras Completas (J. L. Etcheverry, trad., Vol. XVII, pp. 215-252). Buenos Aires, Argentina: Amorrortu.

Freud, S. (2007/1936). Carta a Romain Rolland (Una perturbación del recuerdo en la Acrópolis). En J. Strachey (Ed.), Obras Completas (J. L. Etcheverry, trad., Vol. XXII, pp. 209-222). Buenos Aires, Argentina: Amorrortu.

Freud, S. (2007/1940). La escisión del yo en el proceso defensivo. En J. Strachey (Ed.), Obras Completas (J. L. Etcheverry, trad., Vol. XXIII, pp. 275278). Buenos Aires, Argentina: Amorrortu.

Jaspers, K. (2014/1913). Psicopatología general. México DF, México, Fondo de Cultura Económica.

Lacan, J. (1979/1932). De la psicosis paranoicas en sus relaciones con la personalidad. México: Siglo Veintiuno Editores.

Lacan, J. (2009/1953-1954). El Seminario de Jacques Lacan, Libro 1: Los escritos técnicos de Freud. Buenos Aires, Argentina: Paidós.

Lacan, J. (2012/1958-1959). El Seminario de Jacques Lacan, Libro 6: El deseo y su interpretación. Buenos Aires, Argentina: Paidós.

Lacan, J. (1961-1962). Seminario 9: La identificación. Inédito. Traducción de uso interno EFBA.

Lacan, J. (2009/1962-1963). El Seminario de Jacques Lacan, Libro 10: La angustia. Buenos Aires, Argentina: Paidós.

Lacan, J. (2009/1964). El Seminario de Jacques Lacan, Libro 11: Los cuatro conceptos fundamentales del psicoanálisis. Buenos Aires, Argentina: Paidós.

Lacan, J. (1964-1965). Seminario 12: Problemas fundamentales del psicoanálisis. Inédito. Traducción de uso interno EFBA.

Lacan, J. (2012/1971-1972). Hablo a las paredes. Buenos Aires, Argentina: Paidós. 
López, H. (2011). Lo fundamental de Heidegger en Lacan. Buenos Aires, Argentina: Letra Viva.

Napolitano, G., Basualdo, A., Carbone, N., De Battista, J., Justo, A., Machado, ..., Volta, L. (2008). Clínica y estructura de los fenómenos de despersonalización. La Plata, Argentina: De la Campana.

Shakespeare, W. (2003/1609). Hamlet. Obras Completas (Vol. I, pp. 107-164). Buenos Aires, Argentina: Aguilar. 